\title{
Hepatocellular carcinoma surveillance: the often-neglected practice
}

\author{
Jennifer Sammon ${ }^{1,2}$, Korosh Khalili ${ }^{1,2}$ \\ ${ }^{1}$ Department of Medical Imaging, University of Toronto, Toronto, ON, Canada; ${ }^{2}$ Joint Department of Medical Imaging, University Health Network, \\ Sinai Health System, Women's College Hospital, Toronto, ON, Canada \\ Correspondence to: Korosh Khalili, MD, FRCPC, MHSc. Department of Medical Imaging, University Health Network, Princess Margaret Cancer \\ Centre, 610 University Ave, M5G 2M9, Toronto, ON, Canada. Email: Korosh.khalili@uhn.ca. \\ Comment on: Kanwal F, Singal AG. Surveillance for Hepatocellular Carcinoma: Current Best Practice and Future Direction. Gastroenterology \\ 2019;157:54-64.
}

Submitted Jul 31, 2019. Accepted for publication Aug 05, 2019.

doi: 10.21037/hbsn.2019.08.02

View this article at: http://dx.doi.org/10.21037/hbsn.2019.08.02

Hepatocellular carcinoma (HCC) is the fourth most common cause of cancer worldwide and as such represents a significant global health burden. The clinical societies dedicated to the study of liver diseases all recommend routine surveillance for those at risk. These societies include the Asian-Pacific, European, and American associations for the study of liver disease. The aim of surveillance is to prolong survival, which is to diagnose HCC when the patient remains eligible for potentially curative interventions including resection and transplantation. In a detailed review published recently, Kanwal and Singal discuss the evidence for HCC surveillance, evaluate current surveillance methods and point out new imaging and serological initiatives to improve on current method (1). Their review covers some familiar topics and point to new endeavors in improving the effectiveness of HCC surveillance. In regards to their review article, several important points need to be emphasized as follows.

\section{Promotion of surveillance is the principle challenge}

Worldwide, only small minorities of patients at risk for HCC undergo the recommended surveillance regimen. Japan and Korea remain the only two countries with a national surveillance program. Elsewhere, the utilization of regular ultrasound screening is low, with only $12-29 \%$ of patients diagnosed with HCC receiving regular (annual or biannual) ultrasound surveillance prior to diagnosis (2-4). The poor uptake in surveillance is likely due to a combination of factors including lack of knowledge of guidelines, suboptimal patient adherence to guidelines, healthcare provider bias (alcohol related liver disease) and logistical factors (3). It should be noted that increasing the uptake of surveillance for the atrisk population is much more beneficial than the incremental gain from improving on the current surveillance methods. As healthcare practitioners, we need to advocate for surveillance to our patients, our colleagues, and our public health authorities.

\section{Improvements in ultrasound have increased early HCC detection}

With the advent of harmonic and compound imaging in the mid-2000's, there has been significant increase in the sensitivity of the detection of early HCC (5). The metaanalysis quoted by Kanwal and Singal demonstrating an early HCC detection rate of only $47 \%$ by sonography utilized many studies from pre 2000's (6). However, when the results are analyzed by the decade of publication, there is a significant rise in the detection rate for each decade, rising to $62.2 \%$ for ultrasound alone in the 2010's $(7,8)$. Other studies published over the last decade show that ultrasound alone or with AFP has sensitivity for the detection of early (curable) stage HCC between 69-88\% (5,9-11). For example, in Qidong area of China, biannual ultrasound with AFP caught HCC in early stages in $80.1 \%$ of patients, most of whom had HBV-induced liver disease (12). A recent prospective multicenter trial from France of 1,671 patients with cirrhosis is quite instructive (13). 
Patients with HCC who strictly followed the surveillance recommendations of ultrasound every 6 months were early stage [Barcelona Clinic Liver Cancer (BCLC) stage 0 \& 1 ] in $86 \%$ of cases. They also had statistically higher overall survival. But even those who underwent surveillance less often had early stage tumor in $71 \%$ of cases. The cause of cirrhosis in this population was $\mathrm{HCV}$ in $79 \%$. Therefore Kanwal \& Singal's argument that detection rates may be lower in HCV cirrhosis populations does not hold. The bottom line is that current surveillance recommendations work for the significant majority of patients.

\section{Slow growth rate of early tumor means surveillance gets multiple chances at its detection}

Surveillance of HCC is believed to be effective because in the majority of cases, the progression of the tumor is of sufficiently slow rate and limited extent to allow curative therapies. HCC has a relatively long tumor volume doubling time, with a median of 76.8 days for Hepatitis B virus, 137.2 days for Hepatitis $C$ virus and 99.8 days for non-viral hepatitis (14). The same study has calculated that for a single tumor to grow from 1 to $5 \mathrm{~cm}$ in diameter, the upper limit of "early HCC", it takes a median of 678.9 days. The long tumor volume doubling time allows ultrasound several opportunities to detect the tumor, if it is missed by the initial scan, before it reaches an intermediate or advanced stage (BCLC stage $\mathrm{B}, \mathrm{C}$ and $\mathrm{D}$ ). This improves ultrasound's effective sensitivity. It also explains why studies in which the effectiveness of US surveillance is measured always show better performance than in those when ultrasound is compared to a higher sensitivity modality, such as CT or MRI. In a proportion of patients, CT and MRI detect the tumor at a smaller size before ultrasound has the opportunity to detect it at a later date.

\section{Other imaging means of surveillance are not cost-effective}

Decreased specificity is the cost of increased sensitivity in HCC surveillance. The earlier one attempts to detect tumors, the more likely that a benign nodule is falsely called positive. After all, HCC in the majority of cases occurs with a background of nodular cirrhosis; there are many more benign nodules than malignant ones. False positives result in multiple costly investigations and enhanced follow-up. The decreased specificity, along with the upfront cost of
CT or MRI, is what makes surveillance by these modalities not cost-effective (6). Finding low-risk nodules, so called LI-RADS 2 and 3, on CT and especially MRI scans is quite common in cirrhotic patients and should there be enhanced observation for these, the cost of surveillance would explode. Ultrasound on the other hand is often insensitive to these nodules. Other disadvantages to CT and MRI include limited access, contraindications to contrast, and patient-related factors preventing or reducing scan quality, such as claustrophobia, inability to suspend respiration, and language barriers. Ultrasound is relatively inexpensive, is widely available in all regions of the world and has no contraindications. Radiation is often listed as a disadvantage of $\mathrm{CT}$, but in reality that risk is negligible in patients with cirrhosis in whom median survival is 12 years (15). Imaging based surveillance methods of the future need to overcome these challenges as well as that of cost-effectiveness.

Kanwal \& Singal also discuss serological markers and their potential for use in surveillance in the future. Serological markers in theory may hold many advantages over imaging, including lower cost, higher specificity, ease of application to a wider population, and no contraindications. Unfortunately, AFP is only an adjunct marker and its contribution to ultrasound surveillance is quite incremental in the modern era, likely in the range of $6-8 \%$ improved sensitivity to ultrasound alone (7). Several biomarkers have been identified and tested in preclinical studies including DNA, messenger RNAs, non-coding RNAs, proteins and post-translational protein modifications, with some biomarkers progressing to phase 2 trials. Future research is needed into these biomarkers, but it may allow tailoring of surveillance techniques to those at higher/lower risk of developing HCC in the future (1).

In conclusion, Kanwal and Singal highlight the need for surveillance and the need to improve the uptake of surveillance in those at-risk of developing HCC. It is important to note that there has been a significant change in ultrasound technology over the last few decades and the sensitivity of the detection of HCC in studies published before the mid 2000's is not applicable to today's ultrasound surveillance. Better, more accessible surveillance tools are being developed. But in the meanwhile, far more effort is needed to identify at-risk patients and direct them to surveillance as recommended by current guidelines.

\section{Acknowledgments}

None. 


\section{Footnote}

Conflicts of Interest: The authors have no conflicts of interest to declare.

Ethical Statement: The authors are accountable for all aspects of the work in ensuring that questions related to the accuracy or integrity of any part of the work are appropriately investigated and resolved.

\section{References}

1. Kanwal F, Singal AG. Surveillance for Hepatocellular Carcinoma: Current Best Practice and Future Direction. Gastroenterology 2019;157:54-64.

2. Singal AG, Yopp A, S Skinner C, et al. Utilization of hepatocellular carcinoma surveillance among American patients: a systematic review. J Gen Intern Med 2012;27:861-7.

3. El-Serag HB, Davila JA. Surveillance for hepatocellular carcinoma: in whom and how? Therap Adv Gastroenterol 2011;4:5-10.

4. Thein HH, Campitelli MA, Yeung LT, et al. Improved Survival in Patients with Viral Hepatitis-Induced Hepatocellular Carcinoma Undergoing Recommended Abdominal Ultrasound Surveillance in Ontario: A Population-Based Retrospective Cohort Study. PLoS One 2015;10:e0138907.

5. Khalili K, Menezes R, Kim TK, et al. The effectiveness of ultrasound surveillance for hepatocellular carcinoma in a Canadian centre and determinants of its success. Can J Gastroenterol Hepatol 2015;29:267-73.

6. Tzartzeva K, Obi J, Rich NE, et al. Surveillance Imaging and Alpha Fetoprotein for Early Detection of Hepatocellular Carcinoma in Patients With Cirrhosis: A Meta-analysis. Gastroenterology 2018;154:1706-18.e1.
7. Khalili K. Historical data are not relevant to the diagnostic performance of ultrasound in HCC surveillance. Gastroenterology. doi: 10.1053/j.gastro.2018.12.051.

8. Costentin CE, Layese R, Bourcier V, et al. Compliance With Hepatocellular Carcinoma Surveillance Guidelines Associated With Increased Lead-Time Adjusted Survival of Patients With Compensated Viral Cirrhosis: A MultiCenter Cohort Study. Gastroenterology 2018;155:43142.e10.

9. Kuo YH, Lu SN, Chen CL, et al. Hepatocellular carcinoma surveillance and appropriate treatment options improve survival for patients with liver cirrhosis. Eur J Cancer 2010;46:744-51.

10. Noda I, Kitamoto M, Nakahara H, et al. Regular surveillance by imaging for early detection and better prognosis of hepatocellular carcinoma in patients infected with hepatitis C virus. J Gastroenterol 2010;45:105-12.

11. Santi V, Trevisani F, Gramenzi A, et al. Semiannual surveillance is superior to annual surveillance for the detection of early hepatocellular carcinoma and patient survival. Semin Liver Dis 2017;37:141-51.

12. Chen JG, Zhang YH, Zhu J, et al. Early diagnosis and early treatment for liver cancer in Qidong: survival of patients and effectiveness of screening. Chin J Oncol 2017;39:946-51.

13. Allaire M, Nahon P, Layese R, et al. Extrahepatic cancers are the leading cause of death in patients achieving hepatitis $\mathrm{B}$ virus control or hepatitis $\mathrm{C}$ virus eradication. Hepatology 2018;68:1245-59.

14. An C, Choi YA, Choi D, et al. Growth rate of early-stage hepatocellular carcinoma in patients with chronic liver disease. Clin Mol Hepatol 2015;21:279-86.

15. Zipprich A, Garcia-Tsao G, Rogowski S, et al. Prognostic indicators of survival in patients with compensated and decompensated cirrhosis. Liver Int 2012;32:1407-14.
Cite this article as: Sammon J, Khalili K. Hepatocellular carcinoma surveillance: the often-neglected practice. Hepatobiliary Surg Nutr 2020;9(1):77-79. doi: 10.21037/ hbsn.2019.08.02 